\title{
X-ray Emission from Brown Dwarfs
}

\author{
Thomas Preibisch \\ Max-Planck-Institut für Radioastronomie, Auf dem Hügel 69, D-53121 \\ Bonn, Germany
}

\begin{abstract}
During the last few years, X-ray emission has been detected from numerous brown dwarfs. Most of the X-ray detected brown dwarfs are very young objects with ages of at most $10^{7}$ years, and all are still relatively warm, with late $\mathrm{M}$ spectral types. Their typical fractional X-ray luminosities are $\left(L_{\mathrm{X}} / L_{\mathrm{bol}}\right) \sim 10^{-4}-10^{-3}$, i.e. very similar to the values observed for active very-low mass stars. Their X-ray lightcurves show low-level variability, but in most cases no large flares; this implies that the young brown dwarfs are able to produce quiescent X-ray emission, not only occasional flares. An analysis of the Chandra X-ray spectra of several brown dwarfs yields surprisingly low plasma temperatures between $3 \mathrm{MK}$ and $10 \mathrm{MK}$ for some of the M8-9 dwarfs and indicates a decline in plasma temperature with decreasing effective temperature (or increasing age). The lack of X-ray detections for dwarfs cooler than spectral type M9 is consistent with the strong drop of activity observed in $\mathrm{H} \alpha$ at spectral types around M9. The observed X-ray emission from the young brown dwarfs with late $M$ spectral type can be understood as a consequence of the fact that these objects are still warm enough to maintain partially ionized atmospheres which are capable of sustaining electrical currents. In the cooler, essentially neutral atmospheres of the older $\mathrm{L}$ and $\mathrm{T}$ dwarfs such currents are probably shut off, preventing the buildup of magnetic free energy and the support for magnetically heated chromospheres and coronae.
\end{abstract}

\section{Introduction}

Brown dwarfs (BDs) are objects with masses between $\sim 0.075 M_{\odot}$ (the stellar/substellar boundary) and $\sim 0.012 M_{\odot}$ (the deuterium burning mass limit). In contrast to stars, which derive their luminosity from hydrogen fusion, BDs never reach high enough core temperatures to start hydrogen burning. As they have no sustainable internal fusion energy source, the BDs continuously cool down and dim with time. Only in their very early life, the BDs experience fusion of deuterium. During the first few Myr of their evolution, BDs are much warmer and orders of magnitude brighter than at older ages. Very young $(\sim 1 \mathrm{Myr}$ old $)$ BDs can have spectral types as early as M6.5. As an example for the drastic changes during the early evolution, we note that a $0.03 M_{\odot} \mathrm{BD}$ cools from $2660 \mathrm{~K}$ effective temperature down to $600 \mathrm{~K}$, and its luminosity drops from $\log \left(L_{*} / L_{\odot}\right)=-2.1$ to $\log \left(L_{*} / L_{\odot}\right)=-6.1$, i.e. by 4 orders of magnitude, be- 
tween the ages of $1 \mathrm{Myr}$ and 5 Gyr. Due to their relative brightness young BDs can be much easier detected at larger distances than older BDs. In fact, many young BDs have been discovered in several nearby star forming regions.

Being by definition very cool and dim objects, one would not immediately expect the emission of high energy radiation from BDs. Also, as BDs have a fully convective internal structure, they cannot posses a solar-like $\alpha-\Omega$-dynamo, which is the energy source of the X-ray activity in late-type stars. The discovery of X-ray emission from very-low mass objects was therefore somewhat surprising, and the origin of their activity is still not fully understood.

\section{X-ray detections of brown dwarfs}

The first (and at that time unperceived) detection of X-ray emission from a BD was made as early as 1991, when ROSAT obtained a deep X-ray image of the Chamaeleon star forming region. One of the weak X-ray sources could be identified with a faint ( $V \sim 21 \mathrm{mag}$ ) star-like object, for which no further information was available at that time (recall that the first confirmed BDs were announced in 1995 and see Basri 2000 for a review on observations of BDs). Seven years later, Neuhäuser \& Comeron (1998) presented an optical spectrum of this object, called Cha $\mathrm{H} \alpha 1$, and derived a spectral type of M7.5. Comparison with pre-main sequence evolutionary models (e.g. Baraffe et al. 1998; 2003) showed that this is a sub-stellar object with a mass of $\sim 0.05 M_{\odot}$.

Motivated by this discovery, Neuhäuser et al. (1999) searched for X-ray emission from further BDs with ROSAT (archive) data. Their survey of $26 \mathrm{BDs}$ yielded only one additional tentative detection of a BD in $\rho \mathrm{Oph}$, which however was not confirmed ${ }^{1}$ by Chandra data which provided superior sensitivity and spatial resolution. Although some further detections of BDs could be made with ROSAT, (e.g. Comeron et al. 2000), the X-ray fluxes were in nearly all cases only marginally above the detection limits. Furthermore, the moderate spatial resolution $\left(\sim 15^{\prime \prime}\right)$ of ROSAT often caused identification problems.

The advent of the new X-ray observatories XMM-Newton and especially Chandra boosted the field of X-ray emission from BDs due to the much increased sensitivity and, for Chandra, the superb spatial resolution $\left(\sim 1^{\prime \prime}\right)$. The huge gain in quality of the X-ray data on young clusters is nicely demonstrated by the Chandra observations of IC 348 (Preibisch \& Zinnecker 2001; 2002; Preibisch et al. 1996) and the Orion Nebula Cluster (see Feigelson et al. 2002; 2003 and references therein; Flaccomio et al. 2003): the Chandra data increased the number of X-ray detected cluster members by factors of 2-3 and eliminated essentially all identification problems.

The deep X-ray data on the young cluster IC 348 are of particular interest, because this cluster it is exceptionally well studied at optical and infrared wavelengths, yielding a basically complete spectroscopic identification of all cluster member (see Luhman et al. 2003 and Fig. 1), extending well into the substellar regime. The sample of 288 spectroscopically identified cluster members in IC 348 contains 23 BDs (see Fig. 1). Interestingly, the Chandra data provided

\footnotetext{
${ }^{1}$ The X-ray source seems to be an embedded young star rather than the BD.
} 


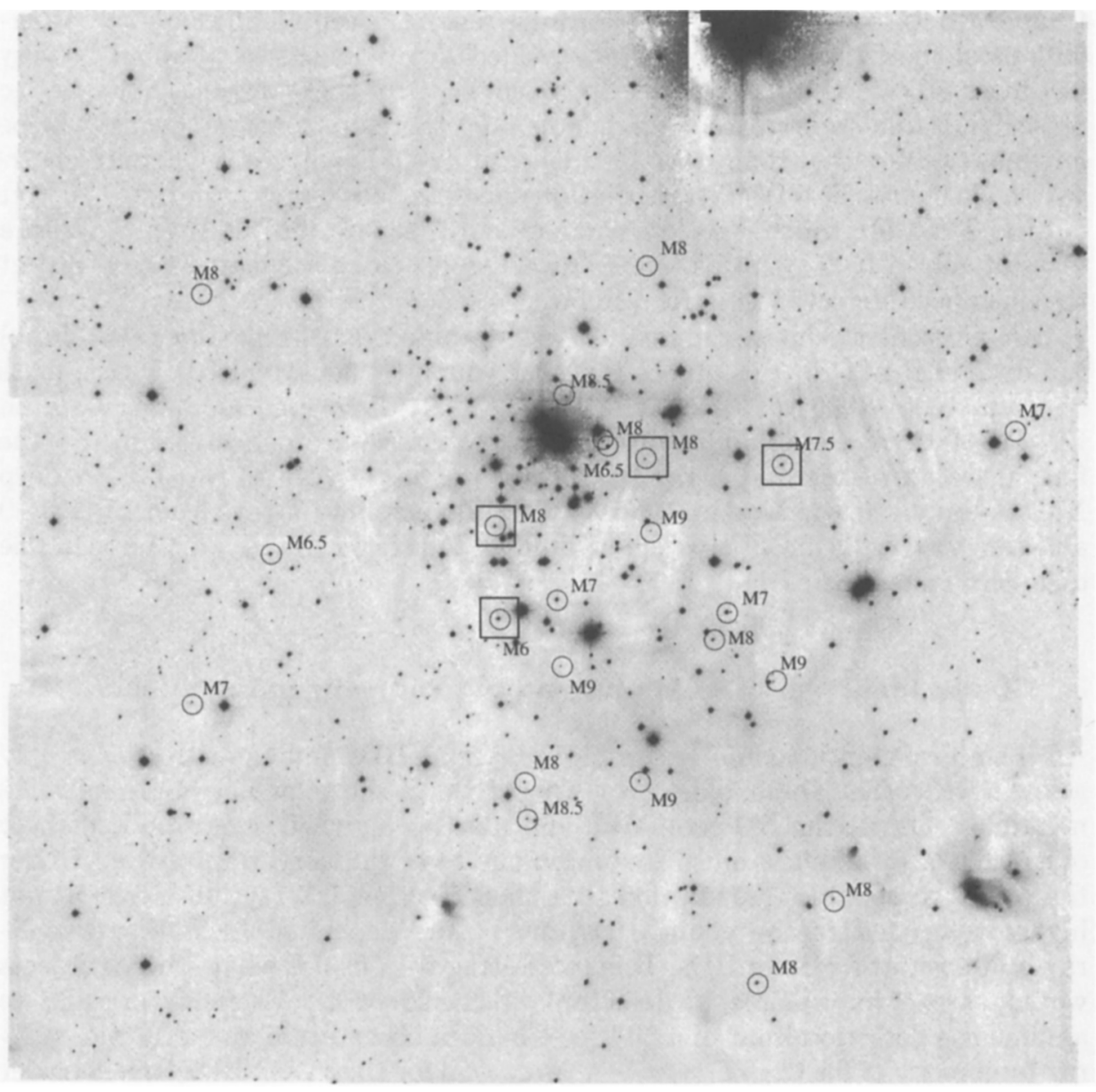

Figure 1. Deep $J$-band mosaic image of IC 348, showing a $18.6^{\prime} \times$ $18.4^{\prime}$ field with a square-root intensity scale; the limiting magnitude of this image is $J \sim 21$. The BDs identified by Luhman et al. (2003) are marked by circles. The four X-ray detected objects (Preibisch \& Zinnecker 2001, 2002) are marked by boxes. 
X-ray detections of 7 very low-mass objects, 4 of which are spectroscopically confirmed BDs (Preibisch \& Zinnecker 2001, 2002). Their X-ray luminosities are around $\sim 10^{28} \mathrm{erg} / \mathrm{sec}$, implying fractional X-ray luminosities in the range $\log \left(L_{\mathrm{X}} / L_{\text {bol }}\right)=-4 \ldots-3$.

$\mathrm{X}$-ray emission from BDs was also discovered in several other star forming regions. Imanishi et al. (2001) detected X-ray emission from two BDs in Chandra data on the $\rho$ Oph star forming region. Deep Chandra X-ray observations of the Orion Nebula Cluster revealed X-ray detections of about 30 very low-mass objects (Feigelson et al. 2003); for most of these objects, however, no optical/infrared spectra are yet available, and therefore some of them might be low-mass stars rather than BDs. Tsuboi et al. (2003) analyzed a Chandra observation of the nearby TW Hya association and detected X-rays from the $\sim 10 \mathrm{Myr}$ old BD TWA-5b, which is a close companion ( 2 " separation) to TWA-5. With a mass of only $\sim 0.02 M_{\odot}$, this M8.5-9 dwarf seems to be the least massive object that has been detected in X-rays so far.

A particularly interesting result was obtained for the nearby $(D=5 \mathrm{pc})$ M9 dwarf LP 944-20; this object is rather young ( $\lesssim 500 \mathrm{Myr}$ ) BD with a mass of about $0.05-0.06 M_{\odot}$. Rutledge et al. (2000) discovered an X-ray flare on LP 944-20 during a Chandra observation, but no quiescent emission. At the flare peak, the fractional X-ray luminosity was $\log \left(L_{\mathrm{X}} / L_{\mathrm{bol}}\right) \sim-3.7$. A deep XMM observation by Martin \& Bouy (2002) detected no X-rays from LP 944-20 and provided a very sensitive upper limit of $\log \left(L_{\mathrm{X}} / L_{\text {bol }}\right)<-6.3$ for possible quiescent emission.

\section{X-ray luminosities of young and old very-low-mass objects}

All the above mentioned detections are for young BDs, mostly with ages of only a few Myr. What about older ( $>1 \mathrm{Gyr}$ ) BDs in the solar neighborhood? As mentioned above, the BD cool down and fade during their evolution and these older BDs are therefore much less luminous than the very young BDs. Under the (quite reasonable) assumption that their fractional X-ray luminosity is not higher than that of the young BDs, one would expect at most very low Xray luminosities for older BDs. It is interesting to consider what kind of objects would, at least in principle, be detectable with current X-ray observatories. If we assume the detection limit of a $50 \mathrm{ksec}$ Chandra observation and a fractional Xray luminosity of $\log \left(L_{\mathrm{X}} / L_{\mathrm{bol}}\right)=-4$, as typical for the young BDs, and consider $\mathrm{BDs}$ at an age of $5 \mathrm{Gyr}$ (from Baraffe et al. 2003), we find the following figures: rather massive BDs $\left(0.07 M_{\odot}\right)$ would be detectable up to distances of $5 \mathrm{pc}$, while objects near the deuterium burning limit $\left(0.015 M_{\odot}\right)$ would be detectable only up to $1.25 \mathrm{pc}$. With the mean number density of BDs in the galactic disk of $\sim 0.1 \mathrm{pc}^{-3}$ (Chabrier 2003), we conclude that the number of X-ray detectable field BDs in the whole sky must be clearly less than 50 , probably less than 10. The prospects of detecting X-ray emission from old field BDs are not very promising,

To put the X-ray properties of the young BDs in perspective, it is interesting to compare them to those of very-low mass stars. A very good basis for the investigation of the X-ray emission from very low-mass objects is the study of Fleming et al. (1995), who presented ROSAT data for a complete, volume 

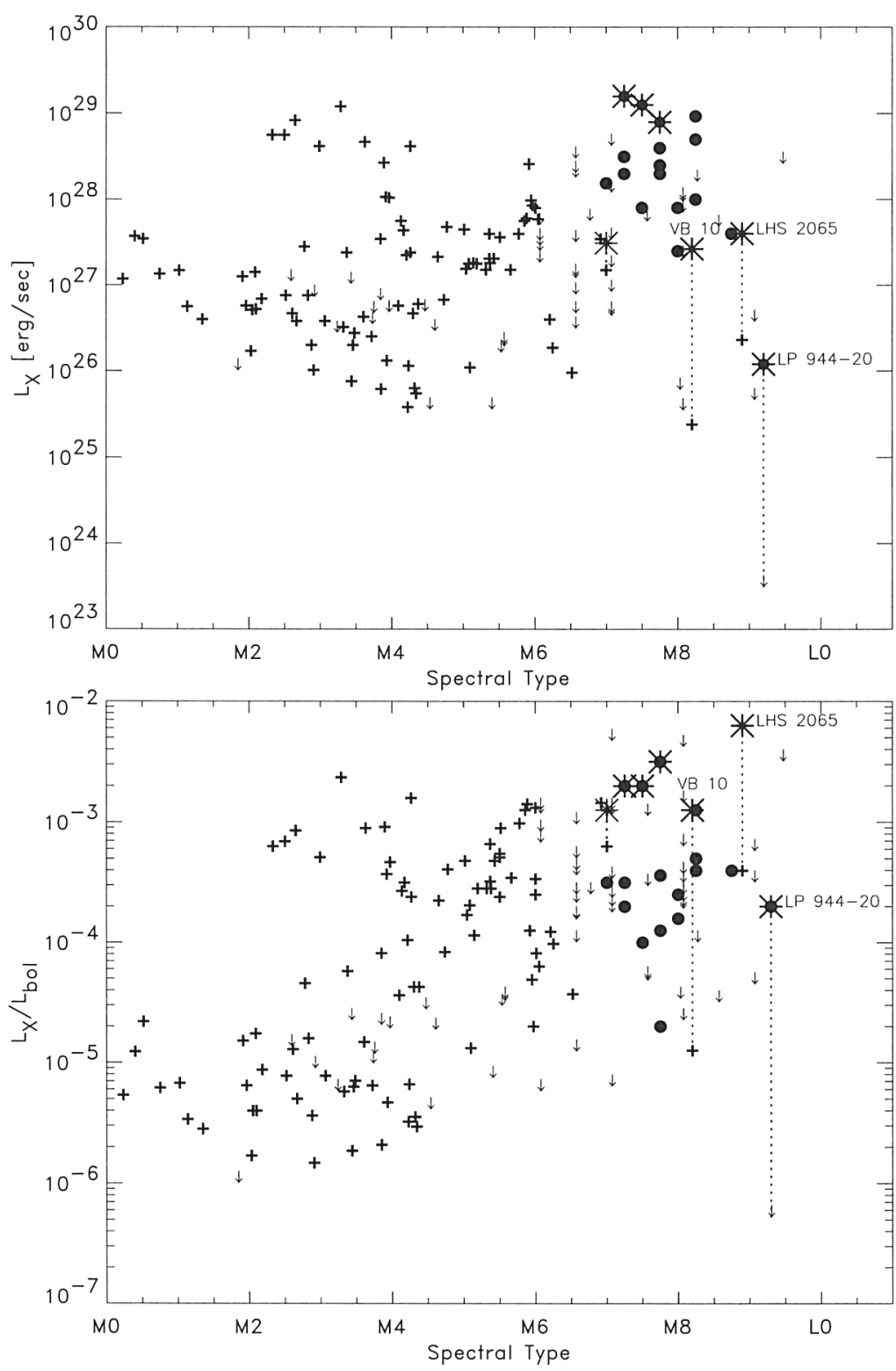

Figure 2. X-ray luminosity (above) and fractional X-ray luminosity (below) versus spectral type. The objects in the complete, volume limited M star sample of Fleming et al. (1995) and some late M stars from Fleming et al. (1993) are shown as crosses. BDs are shown as solid dots. Objects that were flaring during the X-ray observation are indicated with asterisks. For some objects, flaring and quiescent luminosities are connected by dashed lines. 
limited ( $D \leq 7 \mathrm{pc}$ ) sample of M-type dwarfs. The absolute and fractional X-ray luminosities of these objects are shown in Fig. 2, where we also have included data on selected very late type dwarfs from Fleming et al. (1993). Many of the M0 - M7 stars have fractional X-ray luminosities around the saturation limit at $\log \left(L_{\mathrm{X}} / L_{\mathrm{bol}}\right) \sim-3$. Two objects at the cool end of the diagram are of particular interest. One is the M8 star VB 10, which was detected by ROSAT only during a large flare; a recent deep Chandra observation also found evidence for quiescent emission at a level $\log \left(L_{\mathrm{X}} / L_{\text {bol }}\right) \sim-5$, i.e. well below the saturation limit (Fleming et al. 2003). The other is the M9 dwarf LHS 2065, for which Schmitt \& Lieke (2002) detected flaring plus weak quiescent emission in a ROSAT observation; this object currently represents the coolest dwarf with a detection of quiescent $\mathrm{X}$-ray emission, but it is a very-low mass star, just above the bottom of the main-sequence, and not a BD.

Comparing now the young BDs to the older low-mass stars, we find very similar $L_{\mathrm{X}} / L_{\mathrm{bol}}$ ratios at similarly spectral types. This finding suggests that the level of X-ray activity is mainly determined by the effective temperature (i.e. the atmospheric properties) and not (so much) by the mass of the objects.

Another important result is that the coolest objects with $\mathrm{X}$-ray detections are of spectral type M9; no L-type objects have (yet) been detected in X-rays. From the X-ray point-of-view, it is currently unclear whether there really is a change in X-ray activity at spectral type M9, because the few available X-ray data on cooler objects are just not sensitive enough to give useful constraints. However, it is very interesting to note that there is good evidence for a strong change in the chromospheric activity of very cool dwarfs around spectral type M9: their $\mathrm{H} \alpha$ emission, which is usually well correlated to X-ray emission (see e.g. Hawley et al. 1996), shows a clear drop in the activity level $L_{\mathrm{H} \alpha} / L_{\mathrm{bol}}$ just at spectral type M9 - L0 (e.g. Gizis et al. 2000).

\section{Temporal and spectral characteristics of the X-ray emission}

Due to the weak X-ray luminosities of the BDs, the existing X-ray data often consist of only a very small number $(<20)$ of detected photons, too few for detailed spectral or temporal analysis. However, one can take advantage of the superb point-spread function and low background of Chandra, which allows to use very small $\left(1^{\prime \prime}-2^{\prime \prime}\right.$ radius) source extraction regions. Due to the low background, such tiny source regions can usually be expected to contain less than one background photon. All detected photons can then be considered to come from the source, and one can investigate the energies and arrival times of the individual photons to derive at least some information about the characteristics.

Two examples from the Chandra observation of IC 348 are shown in Fig. 3. We first consider the arrival times of the individual detected photons. For L298, the photon arrival times are consistent with a random Poisson process, i.e. suggest that we see quiescent emission at a constant level. In the case of L329, however, the arrival times of the photon clearly cluster around 7 hours after the start of the observation; this suggests a flare-like burst of X-ray emission. A similar analysis for the other X-ray detected BDs shows evidence for flaring X-ray emission in some objects, while other objects show apparently quiescent 

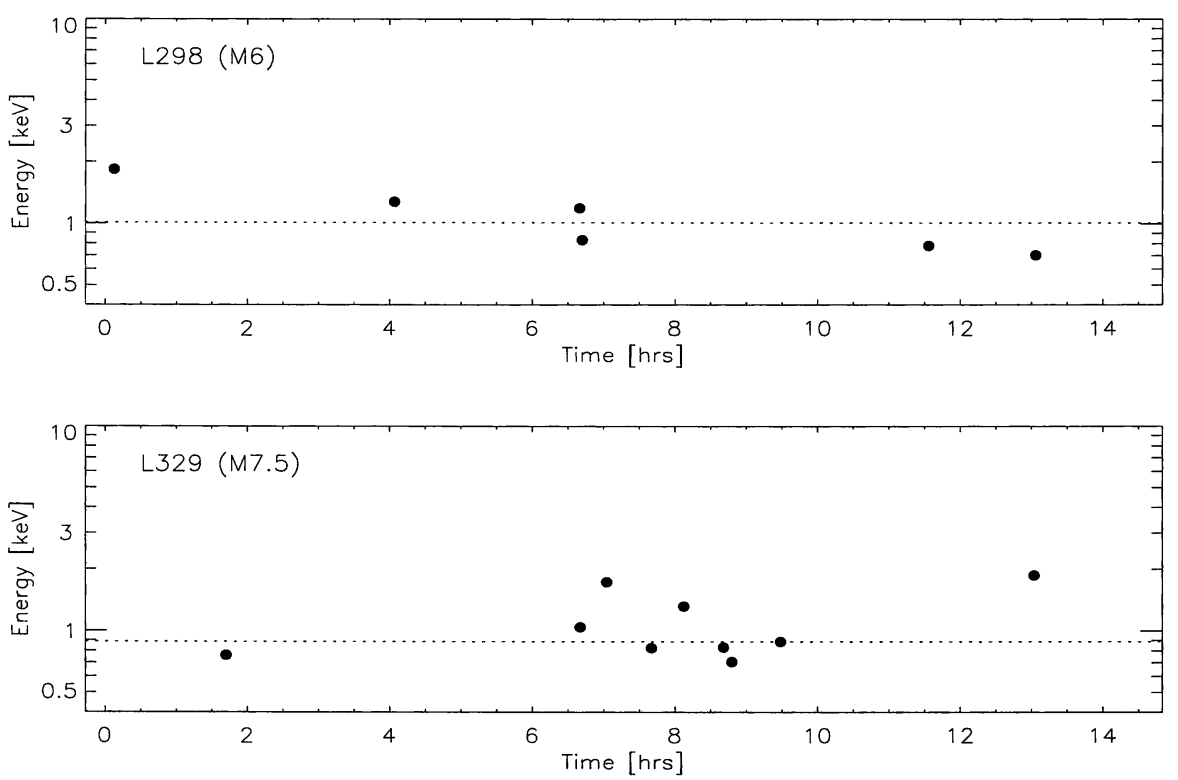

Figure 3. Photon arrival times and energies for the X-ray detected objects L298 and L329 in IC 348. The horizontal dotted lines mark the median photon energies.

emission. The level of X-ray variability seen in the BDs is quite similar to that usually found for coronally active low-mass stars.

Considering now the energies of the individual X-ray photons, we note that only rather soft photons are detected from the two very-low-mass objects shown in Fig. 3. Due to the small number of detected photons, the usual X-ray spectral fitting procedures (spectral binning, background subtraction, and $\chi^{2}$-fitting) cannot be applied for these objects. Nevertheless, even for such faint sources it is sometimes possible to derive useful constraints to the underlying plasma temperature: if small enough source extraction regions are used, no background subtraction is necessary (see above), and then methods like the Cash statistic allow to fit even spectra containing only a handful of photons.

In Fig. 4 we plot the derived X-ray plasma temperatures for several BDs and values for young low-mass stars as a function of their fractional X-ray luminosities and their spectral type. These plots reveal an interesting trend: while the ranges of plasma temperatures found for the BDs $(\sim 3-20 \mathrm{MK})$ and the stars $(\sim 8-50 \mathrm{MK})$ overlap, many of the BDs show values below $10 \mathrm{MK}$ and therefore appear to have systematically cooler plasma temperatures than the stars. As this difference is see for objects with similar fractional X-ray luminosities, it cannot be explained by the corelation between luminosity and temperature generally observed for coronal sources (e.g. Preibisch 1997), and therefore indicates an intrinsic difference between the BDs and stars.

Another interesting trend is seen in the plot of plasma temperature versus spectral type: it seems to suggest a strong decline of plasma temperature at the latest spectral types (M8-9). Particularly surprising are the low plasma 

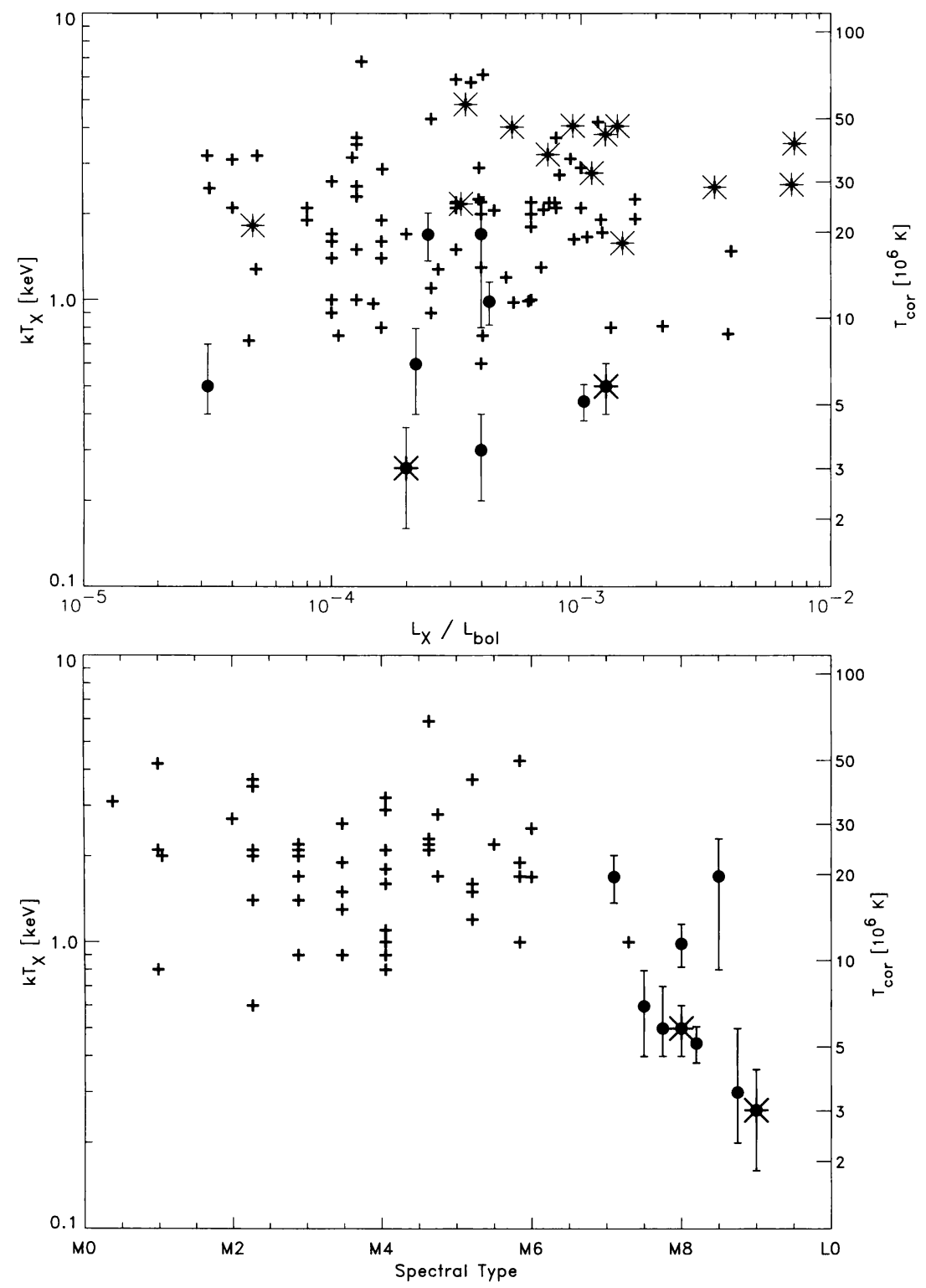

Figure 4. Plasma temperatures versus fractional X-ray luminosity (above) and versus spectral type (below). The crosses mark data for young M-type stars in IC 348 and the Orion Nebula Cluster, the BDs and the very cool dwarf VB10 are shown as big solid dots with error bars. Objects that were flaring during the X-ray observation are indicated with asterisks. References for the data are given in Sections 2 and 3 . 
temperatures found for the X-ray flares on LP 944-20 ( $3 \mathrm{MK})$ and VB 10 $(\sim 5 \mathrm{MK})$; these temperatures are much lower than the values of $\sim 30 \mathrm{MK}$ and more typically found during flares on late-type stars. This suggests that a different flare mechanism is at work in these very-low mass objects than in stars.

\section{Stellar/substellar connections/disconnections}

To make sense of the above results, we group the BDs into different spectral type ranges and finally consider the temporal evolution of the X-ray activity.

\subsection{M6-M8 objects}

The X-ray data suggest that the very young BDs with spectral types M6-M8 show essentially the same signatures of activity as the (older) low-mass (late Mtype) stars. This suggests that the activity is mainly determined by the effective temperature of the dwarfs and not (so much) by their mass. The main difference between BDs and stars, i.e. the presence or absence of central hydrogen burning, seems not to be important for the X-ray activity. This is not so surprising, because it is known since many years that low-mass pre-main sequence stars, which have not yet started central nuclear fusion processes, are generally strong $\mathrm{X}$-ray sources.

The origin of the magnetic activity in young BDs as well as in low-mass pre-main sequence stars is still not exactly known, as both objects classes are fully convective, and therefore the standard solar-like $\alpha-\Omega$ dynamo cannot work. Nevertheless, they are somehow able to generate magnetic fields and sustain a hot corona. Potential alternative dynamo mechanisms are small-scale dynamo action in a highly turbulent convection zone (cf. Giampapa et al. 1996 and references therein) or an $\alpha^{2}$-dynamo, as suggested for example by Küker \& Rüdiger (1999).

The key for understanding the X-ray activity of the very young BDs is the fact that these M6-M8 objects are still warm enough to maintain a partially ionized atmosphere. As the photospheric properties of these young sub-stellar objects are basically the same as those of older stellar objects, it is not surprising that their coronal properties are similar to those of low-mass stars. In other words, the very young BDs "do not yet know" that they are brown dwarfs and not stars, and therefore "behave" like low-mass stars.

\subsection{M8-M9 objects}

The coolest X-ray detected objects have spectral types M8-M9, and there are strong indications that the nature of the X-ray activity is changing in this spectral range. As mentioned above, some of the M8-M9 dwarfs show unusually low $\mathrm{X}$-ray plasma temperatures. Especially striking are the very low plasma temperatures found for the flares on VB 10 and LP 944-20, which strongly suggest that these flares are not caused by the same kind of magnetic reconnection events which are responsible for flares on the Sun and other late-type stars.

It is also interesting to note that the analysis of flaring radio emission from LP 944-20 by Berger et al. (2001) suggested that the magnetic field related to this emission is unusually weak $(B \approx 5 \mathrm{G})$. This is much lower than the typical 
$(\mathrm{kG})$ field strengths associated with flaring emission from late-type stars and indicates that the conditions in the outer atmosphere of this very cool object are significantly different from those of late-type stars.

\subsection{L- and T-dwarfs}

No object cooler than spectral type M9 has up to now been detected to emit Xrays. Although this may be related to observational sensitivity limits, the strong drop in $\mathrm{H} \alpha$ emission around spectral type M9-L0 supports the assumption that the ultra-cool objects really do not show the same kind of activity as late-type stars.

This lack of activity is most likely related to the fact that the atmospheres of objects cooler than $T_{\text {eff }} \sim 2400 \mathrm{~K}$ (corresponding to spectral type M9) are essentially neutral and have a very high electrical resistivity, in which the rapid decay of currents prevents the buildup of magnetic free energy and therefore cannot provide support for magnetically heated chromospheres and coronae (see Fleming et al. 2000). These ultracool dwarfs can therefore not produce the kind of "quiescent" X-ray emission as originates from magnetically confined plasma in the coronae of late-type stars.

However, it is interesting to note that several recent studies found flaring $\mathrm{H} \alpha$ emission in some L- and T-type dwarfs (e.g. Burgasser et al. 2000; Hall 2002; Liebert et al. 2003). These discoveries suggest that even the ultracool dwarfs can show some kind of magnetic activity, which however is probably of different nature than the activity of late-type stars. One possible explanation is that rapidly rising individual flux tubes from the interior of these objects dissipate currents in the atmosphere and cause flares (Mohanty et al. 2002). Further possibilities are discussed in the contribution by G. Basri in these proceedings.

\subsection{Temporal evolution of the $\mathrm{X}$-ray activity}

The evolution of the X-ray activity of BDs can be summarized as follows at the example of a $0.05 M_{\odot}$ BD: At an age of $1 \mathrm{Myr}$, the object is relatively luminous and has a spectral type M7. It emits coronal X-rays at an activity level of $\left(L_{\mathrm{X}} / L_{\text {bol }}\right) \sim 10^{-4}$, which implies an X-ray luminosity of $\sim 8 \times 10^{27} \mathrm{erg} / \mathrm{sec}$, which is rather easily detectable in nearby star forming regions. At an age of $40 \mathrm{Myr}$, its spectral type is M8, and the bolometric luminosity has dropped by a factor of $\sim 12$. Even if the activity level were still $\left(L_{\mathrm{X}} / L_{\mathrm{bol}}\right) \sim 10^{-4}$, its absolute X-ray luminosity would be only $L_{\mathrm{X}} \sim 7 \times 10^{26} \mathrm{erg} / \mathrm{sec}$, which makes it hard to detect for distances above $\sim 90$ pc. After $200 \mathrm{Myr}$, the spectral type is later than M9, its atmosphere is so cool that it gets essentially neutral, and the coronal activity finally ceases.

The future will show whether this relatively simple picture is correct. As the number of known BDs increases rapidly and more and more sensitive observations are performed, we will soon get more insight into the nature of activity in BDs.

\section{References}

Baraffe, I., Chabrier, G., Allard, F., \& Hauschildt, P.H., 1998, A\&A 337, 403 
Baraffe, I., Chabrier, G., Barman, T.S., Allard, F., Hauschildt, P.H. 2003, A\&A 402,701

Basri, G., 2000, ARAA 38, 485

Berger, E., Ball, S., Becker, K.M., et al., 2001, Nat 410, 338

Burgasser, A.J., Kirkpatrick, D., Reid, I.N., et al., 2000, AJ 120, 473

Chabrier, G. 2003, PASP 115, 763

Favata, F., Micela, G., 2003, Space Science Reviews, 108, 577

Feigelson, E.D., \& Montmerle, T., 1999, ARA\&A 37, 363

Feigelson, E.D., Broos, P., Gaffney, J.A., et al., 2002, ApJ 574, 258

Feigelson, E.D., Gaffney, J.A., Gramire, G., et al., 2003, ApJ 584, 911

Flaccomio, E., Damiani, F., Micela, G., et al., 2003, ApJ 582, 398

Fleming, T.A., Schmitt, J.H.M.M., \& Giampapa, M.S., 1995, ApJ 450, 401

Fleming, T.A., Giampapa, M.S., Schmitt, J.H.M.M., Bookbinder, J.A., 1993, ApJ 410, 387

Fleming, T.A., Giampapa, M.S., Schmitt, J.H.M.M., 2000, ApJ 533, 372

Fleming, T.A., Giampapa, M.S., Garza, D., 2003, ApJ 594, 982

Giampapa, M.S., Rosner, R., Kashyap, V., Fleming, T.A., Schmitt, J.H.M.M., \& Bookbinder, J.A., 1996, ApJ 463, 707

Gizis, J.E., Monet, D.G., Reid, I.N., et al., 2000, AJ 120, 1085

Hall, P.B., 2002, ApJ 564, L89

Hawley, S.L., Gizis, J.E., Reid, I.N., 1996, AJ 112, 2799

Imanishi K., Tsujimoto M., Koyama K., 2001, ApJ 563, 361

Küker, M., \& Rüdiger, G., 1999, A\&A 346, 922

Liebert, J., Kirkpatrick, D., Cruz, K.L., et al., 2003, AJ 125, 343

Luhman, K.L., 1999, ApJ 525, 466

Martin, E.L., Bouy, H., 2002, NewAstr 7, 595

Mohanty, S., Basri, G., Shu, F., Allard, F., Chabrier, G. 2002, ApJ 571, 469

Najita, J.R., Tiede, G.P., \& Carr, J.S., 2000, ApJ 541, 977

Neuhäuser, R., 1997, Science 267, 1363

Neuhäuser, R., Comeron, F., 1998, Science 282, 83

Neuhäuser, R., Briceno C., Comeron, F., 1999, A\&A 343, 883

Preibisch Th., 1997, A\&A 320, 525

Preibisch Th., Zinnecker H., 2001, AJ 122, 866

Preibisch, Th., \& Zinnecker H. 2002, AJ 123, 1613

Preibisch, Th., Zinnecker H., Herbig, G.H. 1996, A\&A 310, 456

Rutledge, R.E., Basri, G., Martin, E.L., \& Bildsten, L., 2000, ApJ 538, L141

Schmitt, J.H.M.M., Liefke C., 2002, A\&A 382, L9

Tsuboi, Y., Maeda Y., Feigelson, E.D., et al. 2003, ApJ 587, L51 